\title{
Effects of Bauhinia championii (Benth.) Benth. polysaccharides on the proliferation and cell cycle of chondrocytes
}

\author{
LIANGLIANG CAI, HONGZHI YE, FANGRONG YU, HUITING LI, JIASHOU CHEN and XIANXIANG LIU \\ Academy of Integrative Medicine, Fujian University of Traditional Chinese Medicine, Fuzhou, Fujian 350108, P.R. China
}

Received November 6, 2012; Accepted February 8, 2013

DOI: $10.3892 / \mathrm{mmr} .2013 .1368$

\begin{abstract}
It has been recently shown that polysaccharides isolated from plants exhibit a number of beneficial therapeutic properties. Bauhinia championii (Benth.) Benth. has been widely used for the clinical treatment of knee osteoarthritis (OA) in China. However, the underlying molecular mechanisms of knee OA treatment have yet to be elucidated. In the present study, we investigated the effects of Bauhinia championii (Benth.) Benth. polysaccharides (BCBPs) on the proliferation and cell cycle of chondrocytes on 4-week-old male Sprague Dawley rats. Immunohistochemical staining was used to identify chondrocytes and an MTT assay was used to evaluate cell viability. Flow cytometry was used for cell cycle analysis. The mRNA and protein expression levels of cyclin D1, CDK4 and CDK6 in chondrocytes were detected using reverse transcription polymerase chain reaction (RT-PCR) and western blot analysis, respectively. The data demonstrate that BCBP treatment increased the viability of chondrocytes. In addition, BCBP treatment reduced the cell population in the $\mathrm{G} 0$ / G1 phase, whereas the cell population was increased in the $\mathrm{S}$ phase. Furthermore, BCBP treatment enhanced the expression of cyclin D1, CDK4 and CDK6. These results indicate that $\mathrm{BCBP}$ treatment promotes cell proliferation by accelerating the G1/S transition.
\end{abstract}

\section{Introduction}

Osteoarthritis (OA) is a joint disease, characterized by the degeneration of articular cartilage and bone remodeling

Correspondence to: Professor Xianxiang Liu, Academy of Integrative Medicine, Fujian University of Traditional Chinese Medicine, 1 Huatuo Road, Minhou Shangjie, Fuzhou, Fujian 350108, P.R. China

E-mail: liuxianxiang@163.com

Abbreviations: BCBP, Bauhinia championii (Benth.) Benth. polysaccharide; OA, osteoarthritis; DMSO, dimethyl sulfoxide; MTT, 3-(4,5-dimethythiazol-2-yl)-2,5-diphenyltetrazolium bromide

Key words: Bauhinia championii (Benth.) Benth., polysaccharide, proliferation, cell cycle, chondrocyte that clinically results in pain and joint stiffness, which involves mechanical and biological factors (1,2). Adult articular cartilage consists of a relatively sparse population of non-proliferating chondrocytes which produce a large amount of extracellular matrix (ECM), mainly composed of two major types of macromolecules, collagens (types II, IX and XI) and proteoglycans. Chondrocytes are responsible for tissue homeostasis, including the synthesis and degradation of ECM components (3). Therefore, chondrocyte proliferation is important in maintaining cellular function.

The cell cycle represents a highly regulated series of events that leads to eukaryotic cell reproduction. During the early stages of the cell cycle, DNA is replicated and the chromosomes are duplicated during the transition through to the $\mathrm{S}$ phase. This process begins at specific DNA sites known as replication origins. At these sites, the DNA replication licensing machinery opens the DNA double helix, exposing it to enzymes that conduct DNA synthesis (4). The S phase is followed by chromosomal segregation and nuclear and cell division, which is collectively termed the $\mathrm{M}$ phase. The majority of cell cycles contain additional gap phases between the G1 and S phase, which provide more time for growth and also serve as important regulatory transitions, during which progression to the next cell cycle stage is controlled by intracellular and extracellular signals $(5,6)$. G1 is a particularly important regulatory period, since it is during this phase that most cells either become committed to continued division or exit from the cell cycle $(7,8)$.

The destiny of a cell is strictly controlled by a set of cell cycle factors. In particular, the Ser/Thr protein kinases CDK4 and CDK6 are important for cell cycle progression at the G1 phase. Cyclin D1 is also a positive regulator of the G1/S transition and functions as one of the key restriction points in the cell cycle by binding to CDK4 or CDK6 to control cell cycle progression from the $\mathrm{G} 1$ to the $\mathrm{S}$ phase (9).

Bauhinia championii (Benth.) Benth. polysaccharide (BCBP) treatment promotes chondrocyte proliferation and may be associated with the upregulation of cyclin D1, CDK4 and CDK6 levels, which are important targets in the G1/S transition of the cell cycle.

Natural products have been considered as alternative medicine for several years. Numerous plants and their constituents have been shown to possess beneficial therapeutic effects for various diseases (10). Polysaccharides isolated from plants have attracted a large amount of research attention due to 
their broad spectrum of therapeutic properties and their relatively low toxicity (11-13). In modern pharmacological studies, polysaccharides isolated from plants have been shown to carry out anti-inflammatory, anti-oxidant and antitumor activities (14-16). However, to the best of our knowledge, no previous studies have been conducted on the effects of BCBP on chondrocytes.

In the present study, polysaccharides from Bauhinia championii (Benth.) Benth. were obtained using water extraction and ethanol precipitation. To investigate the effects of BCBP on the proliferation and cell cycle of chondrocytes, an MTT assay was used to evaluate the proliferative activity of BCBP. In addition, the cell cycle distribution was detected by flow cytometry. Furthermore, the mRNA and protein expression levels of cyclin D1, CDK4 and CDK6 were determined using reverse transcription polymerase chain reaction (RT-PCR) and western blot analysis, respectively.

\section{Materials and methods}

Drug preparation. Bauhinia championii (Benth.) Benth. was ground and dried and the powder $(100 \mathrm{~g})$ was extracted with pure water $(1: 15, \mathrm{w} / \mathrm{v})$ at $85^{\circ} \mathrm{C}$ for $6 \mathrm{~h}$, followed by centrifugation at 3,000 r/min for $15 \mathrm{~min}$. Water extracts were collected and the dregs extracted. The combined extracts were pooled and condensed to $\sim 100 \mathrm{ml}$ under a reduced pressure. Subsequently, $400 \mathrm{ml}$ of $95 \%$ alcohol by volume was slowly added by stirring to precipitate the polysaccharides. This polysaccharide sediment was further refined by repeated dissolution and precipitation 3 times, followed by a wash with ethanol, acetone and ether (17).

Animals. Healthy and clean 4-week-old male Sprague-Dawley rats $(n=30$; weight, 90-110 g) were purchased from the Shanghai Laboratory Animal Commission (SLAC, Shanghai, China). The animal license number for the rats was SCXK (Shanghai) 2008-0005. Experiments involving the animals complied with the Guidance Suggestions for the Care and Use of Laboratory Animals 2006 administered by the Ministry of Science and Technology of the People's Republic of China (18).

Reagents. Fetal bovine serum (FBS), Dulbecco's modified Eagle's medium (DMEM) and Trypsin-EDTA were obtained from HyClone (Logan, UT, USA). Type II collagenase and 3-(4,5-dimethy thiazol-2-yl)-2,5-diphenyltetrazolium bromide (MTT) were obtained from Sigma-Aldrich (St. Louis, MO, USA). The TRIzol reagent was obtained from Invitrogen (Carlsbad, CA, USA). A reverse transcription test kit was obtained from Promega (Madison, WI, USA) and the cell cycle test kit was purchased from Becton-Dickinson (San Jose, CA, USA). A total protein extraction kit was purchased from Nanjing KeyGen Biotech Co., Ltd. (Nanjing, China). Polyvinylidene fluoride (PVDF) membranes were purchased from Millipore (Billerica, MA, USA). Rabbit anticyclin D1, -CDK4, -CDK6 and - $\beta$-actin and HRP secondary goat anti-rabbit antibodies were purchased from Santa Cruz Biotechnology, Inc. (Santa Cruz, CA, USA). Any additional chemicals, unless otherwise stated, were obtained from Sigma-Aldrich.
Isolation and culture of rat chondrocytes. Articular cartilage obtained from rat knee joints was rinsed in PBS and DMEM three times. The cartilage was sectioned into $1-\mathrm{mm}^{3}$ slices, digested with $0.2 \%$ type II collagenase and then transferred to a $37^{\circ} \mathrm{C}$ incubator to isolate the chondrocytes. The supernatant was collected every $2 \mathrm{~h}$ and centrifuged at 1,000 r/min for 5 min to obtain the cell pellet. The cells were then filtered through 200 mesh stainless steel filters, seeded (5x10 5 cells/ $\mathrm{ml}$ ) in 6-well plates in DMEM containing $10 \%$ FBS and cultivated in a $\mathrm{CO}_{2}$ incubator $\left(5 \% \mathrm{CO}_{2}\right.$ at $\left.37^{\circ} \mathrm{C}\right)(19)$. The primary cultured cells were observed under an inverted microscope and passaged upon reaching $80 \%$ confluence.

Identification of chondrocytes using immunohistochemical staining. The second generation chondrocytes were seeded onto cover slips and cultured for $72 \mathrm{~h}$. The cells were washed with PBS and fixed in $4 \%$ neutral formalin for $30 \mathrm{~min}$. Subsequent steps were performed according to the manufacturer's instructions. The expression of type II collagen was observed using immunohistochemical staining. Images were captured at a magnification of $\mathrm{x} 40$.

Evaluation of cell viability using the MTT assay. The viability of chondrocytes was assessed using the MTT colorimetric assay. The second passage chondrocytes were seeded in 96-well plates at a density of $1.0 \times 10^{4}$ cells/well in $0.1 \mathrm{ml}$ of $10 \% \mathrm{FBS} /$ DMEM. The chondrocytes were treated with various concentrations of BCBP for 24,48 or $72 \mathrm{~h}$. The medium was then removed and $20 \mu \mathrm{l}$ of $0.5 \% \mathrm{MTT}$ solution was added to each well, followed by incubation at $37^{\circ} \mathrm{C}$ for $4 \mathrm{~h}$. The purple-blue MTT formazan precipitate was dissolved in $100 \mu$ l dimethyl sulfoxide (DMSO). The absorbance was measured at $490 \mathrm{~nm}$ using an ELISA reader (ELx800 ${ }^{\mathrm{TM}}$; BioTek Instruments, Inc., Winooski, VT, USA).

Cell cycle analysis using flow cytometry with PI staining. Chondrocytes were seeded in 35-mm petri dishes at a density of $5 \times 10^{4}$ cells $/ \mathrm{ml}$. The chondrocytes were treated with various concentrations of BCBP for $48 \mathrm{~h}$. The cells were then digested, collected and resuspended in PBS. The cell concentration was adjusted to $1 \times 10^{6}$ cells $/ \mathrm{ml}$ following centrifugation. Solutions A, B and C were added according to the manufacturer's instructions. ModFit software was used to analyze the DNA content and to count the cell numbers in the G0/G1, $\mathrm{S}$ and $\mathrm{G} 2 / \mathrm{M}$ phases.

RNA extraction and RT-PCR analysis. Chondrocytes $\left(1 \times 10^{5}\right)$ were seeded in 6-well plates in $2 \mathrm{ml}$ of medium and treated with various concentrations of BCBP for $48 \mathrm{~h}$. Total RNA was isolated with TRIzol reagent. RNA $(1 \mu \mathrm{g})$ was reverse transcribed into cDNA. The obtained cDNA was used to determine the mRNA levels of cyclin D1, CDK4 and CDK6. $\beta$-actin was used as an internal control. The sequences of the primers used for amplification of cyclin D1, CDK4 and CDK6 were as follows: Cyclin D1 forward, 5'-AAT GCC AGA GGC GGA TGA GA-3' and reverse, 5'-GCT TGT GCG GTA GCA GGA GA-3', 189 bp; CDK4 forward, 5'-GAA GAC GAC TGG CCT CGA GA-3' and reverse, 5'-ACT GCG CTC CAG ATT CCT CC-3', 109 bp; CDK6 forward, 5'-TTG TGA CAG ACA TCG ACG AG-3' and reverse, 5'-GAC AGG TGA GAA TGC 

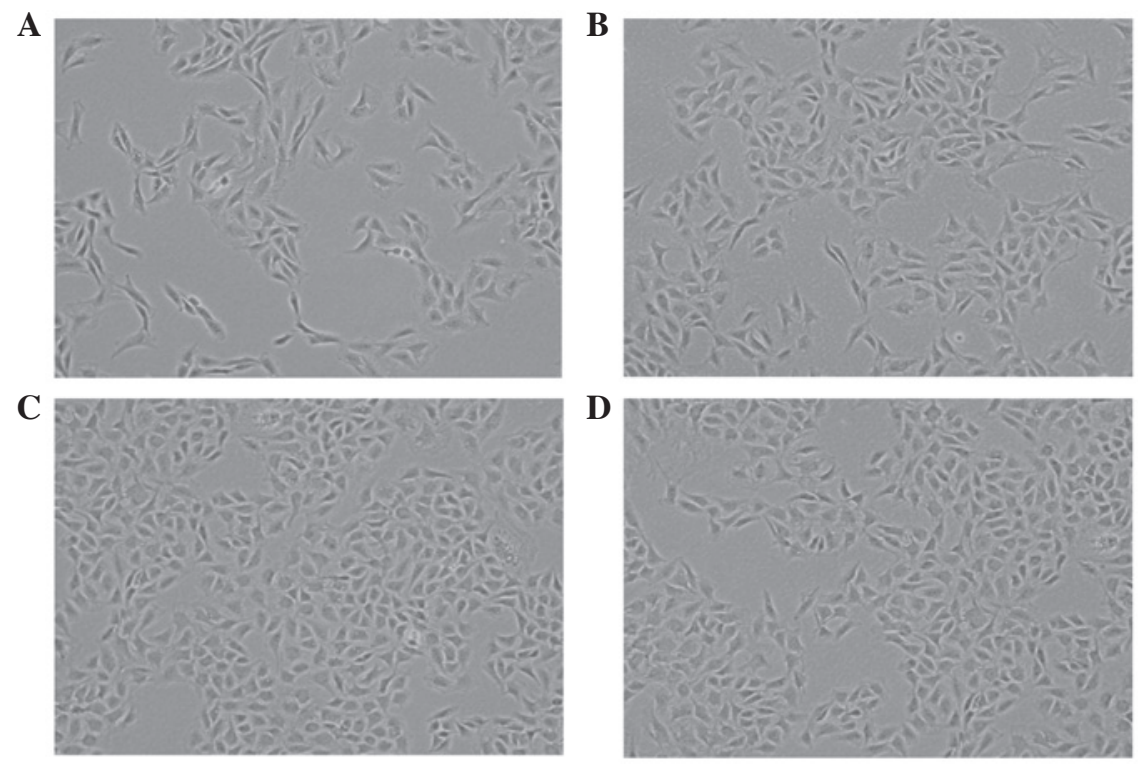

Figure 1. Effect of BCBP on the morphological changes of chondrocytes. (A) Untreated control chondrocytes; (B-D) chondrocytes treated with 50, 100 and $200 \mu \mathrm{g} / \mathrm{ml} \mathrm{BCBP}$, respectively, for $48 \mathrm{~h}$. The morphological changes of chondrocytes were observed using phase contrast microscopy. Images were captured at a magnification of x100. BCBP, Bauhinia championii (Benth.) Benth. polysaccharide.
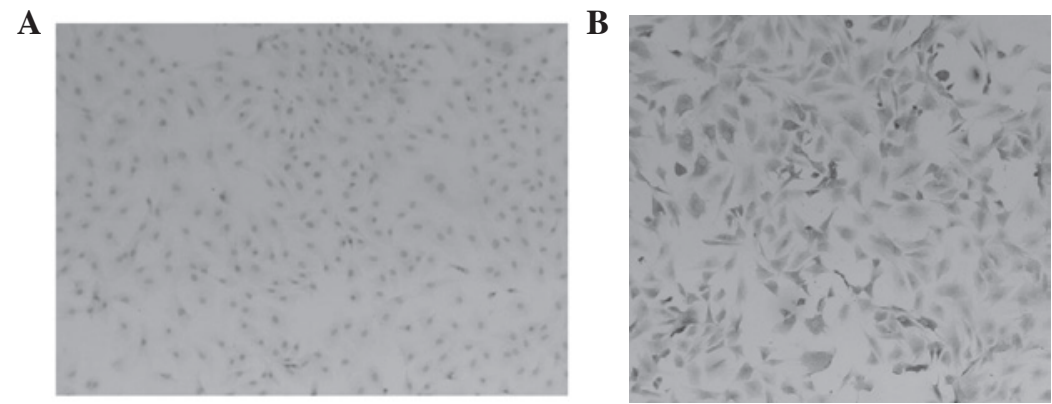

Figure 2. Identification of chondrocytes. Immunohistochemical staining demonstrated that (A) the cytoplasm of the negative group of chondrocytes was unstained, whereas (B) the cytoplasm of chondrocytes was stained. Images were captured at a magnification of $\mathrm{x} 40$.

AGG TT-3', $151 \mathrm{bp}$; and $\beta$-actin forward, 5'-CGT TGA CAT CCG TAA AGA CC-3' and reverse, 5'-GGA GCC AGG GCA GTA ATC T-3', 108 bp.

Western blot analysis. Chondrocytes were seeded in culture flasks and treated with or without $\mathrm{BCBP}$ at $37^{\circ} \mathrm{C}$ for $48 \mathrm{~h}$. Cells were scraped from the culture, washed twice with PBS and then suspended in $30 \mu \mathrm{l}$ western blotting lysis buffer. The protein concentration was determined using a bicinchoninic acid (BCA) protein assay. Samples were loaded with $20 \mu \mathrm{g}$ protein and separated by electrophoresis on $12 \%$ SDS-polyacrylamide gels. Following electrophoresis, protein blots were transferred to PVDF membranes. The membranes were blocked with $5 \%$ skimmed milk in TBST solution and incubated overnight with the primary antibodies at $4^{\circ} \mathrm{C}$. The membranes were then washed in TBST and exposed to secondary antibodies. The membranes were developed by ECL Plus Western Blotting Detection Reagents (Molecular Imager ChemiDoc XRS System; Bio-Rad, Hercules, CA, USA)

Statistical analysis. Data were presented as the mean \pm standard deviation (SD) when appropriate. All the experiments were repeated at least three times and the representative results are shown. Results were analyzed by one-way ANOVA (control vs. treatments) followed by Student's t-test using SPSS 16.0 software. $\mathrm{P}<0.05$ was considered to indicate a statistically significant difference.

\section{Results}

Morphological observation and characterization of chondrocytes. The second generation chondrocytes were treated with various concentrations of BCBP for $48 \mathrm{~h}$. BCBP treatment significantly increased the total number of chondrocytes compared with the untreated control cells (Fig. 1), indicating that BCBP promotes chondrocyte proliferation.

To characterize the chondrocytes, the effects of BCBP on type II collagen expression were evaluated using immunohistochemical staining. Type II collagen matrix is a chondrocyte-specific protein and may be used as a biomarker to identify chondrocytes $(20,21)$. The results demonstrated that the cytoplasm of the negative group of cells was unstained (Fig. 2A), whereas the cytoplasm of chondrocytes was stained a brown-yellow color (Fig. 2B). 

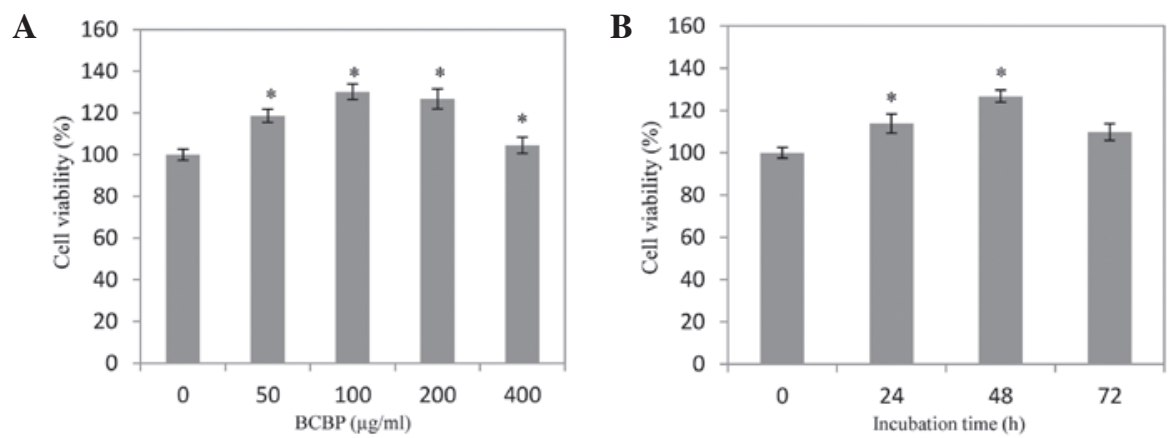

Figure 3. Effect of BCBP on the viability of chondrocytes. Chondrocytes were treated with (A) the indicated concentrations of BCBP for $48 \mathrm{~h}$ and (B) with $100 \mu \mathrm{g} / \mathrm{ml}$ of BCBP for 24,48 or $72 \mathrm{~h}$. The viability of chondrocytes was determined using the MTT assay. Data were normalized to the viability of untreated cells $(100 \%)$. Data are presented as the average \pm SD (error bars). " $\mathrm{P}<0.05$, significant vs. untreated cells. BCBP, Bauhinia championii (Benth.) Benth. polysaccharide; SD, standard deviation.

A

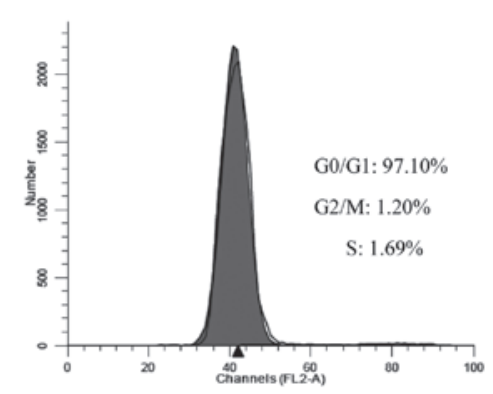

C

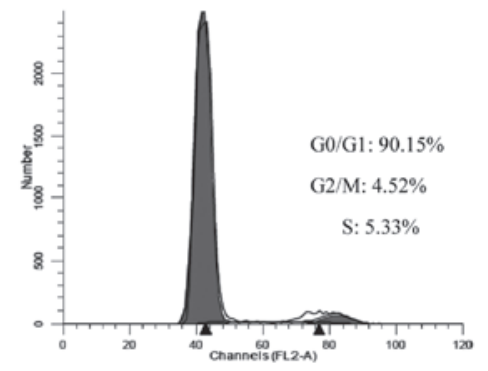

$\mathbf{E}$

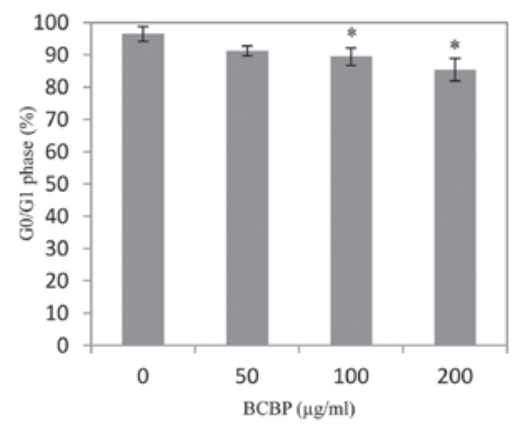

B

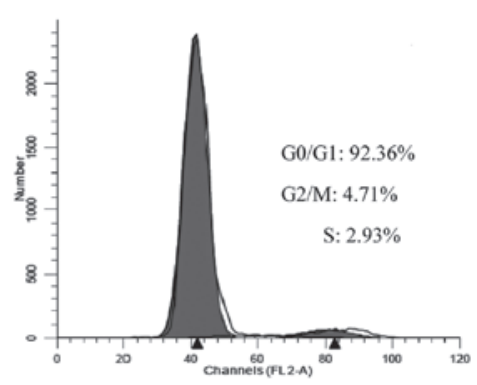

D

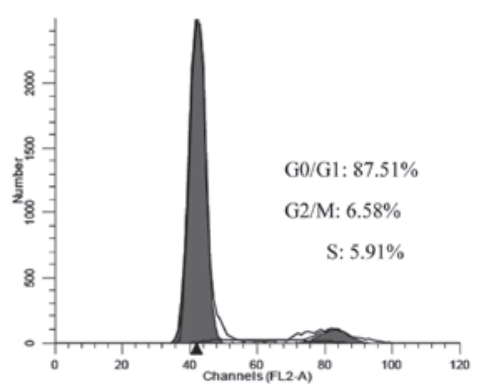

F

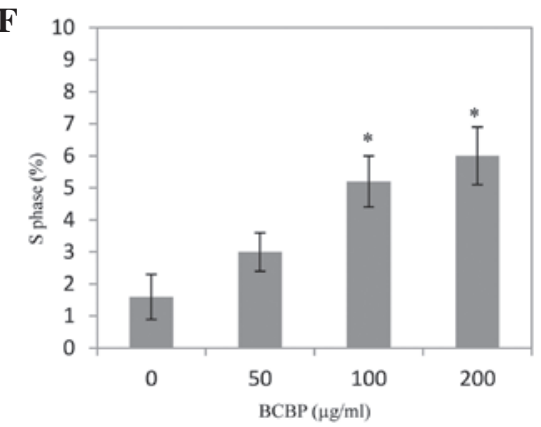

Figure 4. Effect of BCBP on the cell cycle progression of chondrocytes. Following treatment with BCBP, chondrocytes were stained with PI and analyzed using flow cytometry. (A) Untreated control chondrocytes; (B-D) chondrocytes treated with 50, 100 and $200 \mu \mathrm{g} / \mathrm{ml}$ of BCBP, respectively. The percentage of chondrocytes in the (E) G0/G1 and (F) S phases following BCBP treatment. Data are presented as the average \pm SD (error bars) from three independent experiments. "P<0.05 compared with control cells. BCBP, Bauhinia championii (Benth.) Benth. polysaccharide; SD, standard deviation.

Effect of BCBP on the viability of chondrocytes. The cells were treated with $50-200 \mu \mathrm{g} / \mathrm{ml}$ BCBP for $48 \mathrm{~h}$ to evaluate the effect of BCBP on the viability of chondrocytes. As shown in Fig. 3, the viability of chondrocytes was increased by $18.72 \pm 2.61$, $30.13 \pm 3.24$ and $26.87 \pm 3.77 \%$ when the cells were treated with 50,100 and $200 \mu \mathrm{g} / \mathrm{ml}$ of BCBP, respectively. Furthermore, the viability of chondrocytes was increased by $3.24 \pm 2.06$, $11.57 \pm 2.30$ and $19.73 \pm 2.42 \%$ when the cells were treated with
$200 \mu \mathrm{g} / \mathrm{ml} \mathrm{BCBP}$ for 24,48 and $72 \mathrm{~h}$, respectively, compared with untreated cells $(\mathrm{P}<0.05)$.

Effect of BCBP on the cell cycle of chondrocytes. Chondrocytes were treated with 50,100 and $200 \mu \mathrm{g} / \mathrm{ml}$ of BCBP for $48 \mathrm{~h}$ to explore the effect of $\mathrm{BCBP}$ on cell cycle progression. As shown in Fig. 4, BCBP treatment decreased the percentage of cells in the G0/G1 phase, while the percentage of cells in the 
A

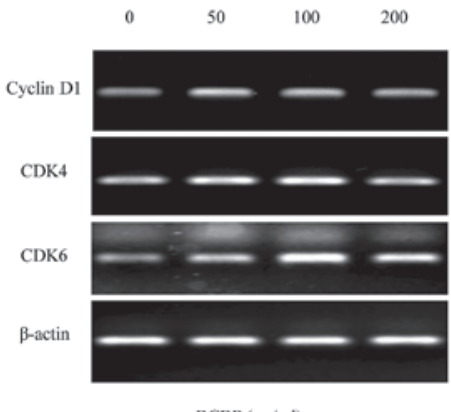

C

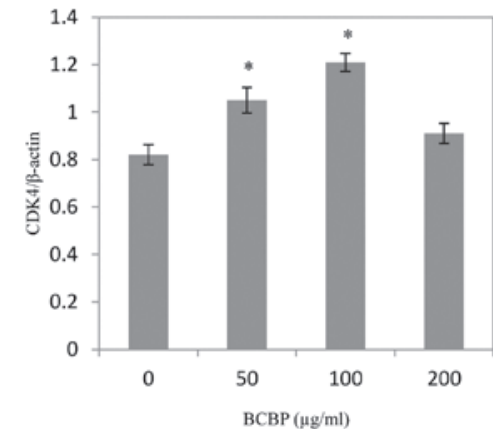

B

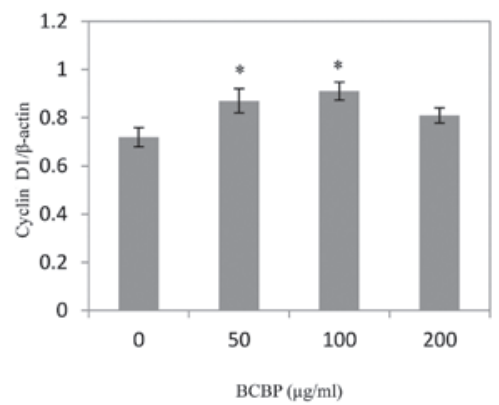

D

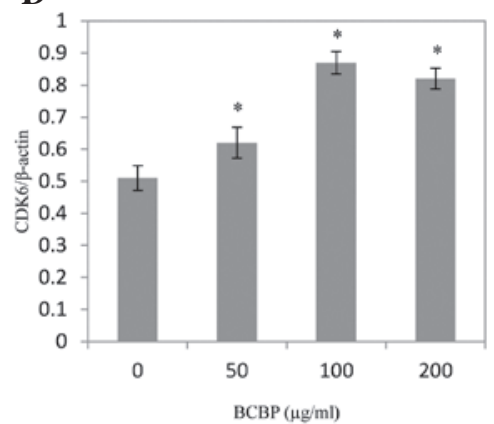

Figure 5. Effect of BCBP on the mRNA expression of cyclin D1, CDK4 and CDK6 in chondrocytes. (A) The mRNA expression levels of cyclin D1, CDK4 and CDK6 were analyzed using RT-PCR. $\beta$-actin was used as an internal control. The mRNA expression of (B) cyclin D1, (C) CDK4 and (D) CDK6 in BCBP-treated and untreated cells. For the quantification of RT-PCR analysis, data are presented as the average \pm SD (error bars). ${ }^{*} \mathrm{P}<0.05$, significant vs. untreated cells. BCBP, Bauhinia championii (Benth.) Benth. polysaccharide; RT-PCR, reverse transcription polymerase chain reaction; SD, standard deviation.
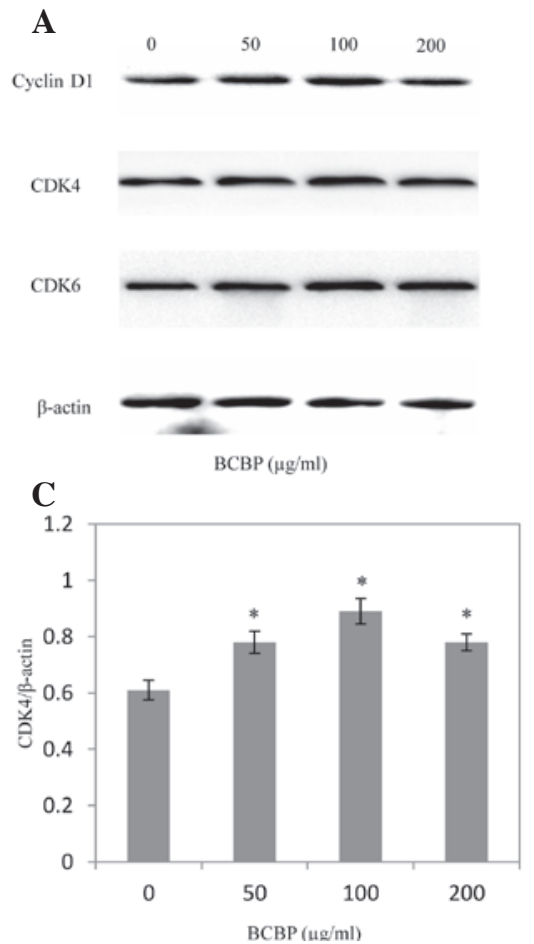

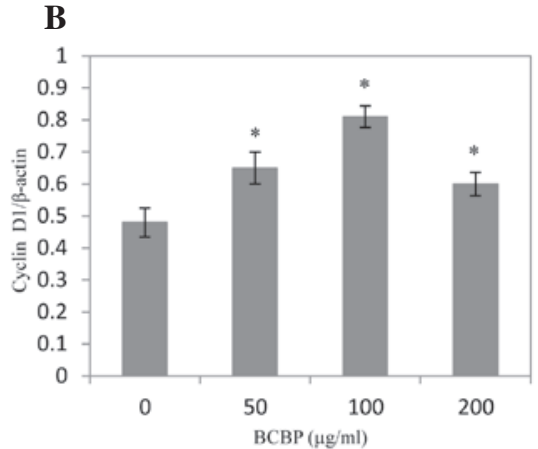

D

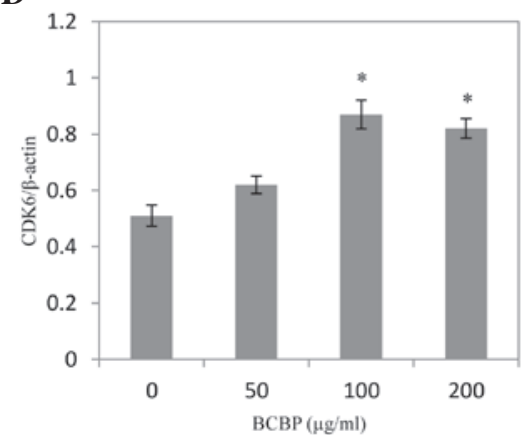

Figure 6. Effect of BCBP treatment on the protein expression levels of cyclin D1, CDK4 and CDK6 in chondrocytes. (A) The protein expression levels of cyclin D1, CDK4 and CDK6 were analyzed using western blot analysis. $\beta$-actin was used as an internal control. The protein expression levels of (B) cyclin D1, (C) CDK4 and (D) CDK6 in BCBP-treated and untreated cells. For the quantification of western blot analysis, data are presented as the average \pm SD (error bars). $\mathrm{P}<0.05$, significant vs. untreated cells. BCBP, Bauhinia championii (Benth.) Benth. polysaccharide; SD, standard deviation.

$\mathrm{S}$ phase was increased $(\mathrm{P}<0.05)$. Taken together, the results suggest that BCBP enhances cell cycle progression of the chondrocytes by promoting the G1 to S phase transition.
Effect of $B C B P$ on the expression of cyclin D1, CDK4 and CDK6 in chondrocytes. Following BCBP treatment for $48 \mathrm{~h}$, the mRNA and protein expression levels of cyclin D1, CDK4 
and CDK6 were determined using RT-PCR and western blot analysis, respectively. The results of the RT-PCR assay showed that BCBP treatment significantly increased cyclin D1, CDK4 and CDK6 mRNA expression levels in chondrocytes $(\mathrm{P}<0.05$, Fig. 5). The protein expression patterns of cyclin D1, CDK4 and CDK6 were similar to those of their respective mRNA levels $(\mathrm{P}<0.05$, Fig. 6).

\section{Discussion}

OA, the most common age-related cartilage and joint disorder, is a slow progressing degenerative disease characterized by the degradation of the ECM and cell death resulting in a gradual loss of articular cartilage integrity (22-24). The only cell type present in mature cartilage is chondrocytes, which are responsible for repairing damaged cartilage tissue.

Low proliferative activity in osteoarthritic chondrocytes has recently been demonstrated; therefore, a treatment that promotes the proliferative activity of chondrocytes may be efficient to delay or even cease the progression of OA (25). The present study mainly focused on exploring drugs that promote the proliferation of chondrocytes.

Bauhinia championii (Benth.) Benth. is a herbal medicine demonstrated to be clinically effective in treating OA. It possesses the functions of expelling wind and removing dampness, in addition to promoting blood circulation and relieving pain. However, the mode of action of this treatment for OA remains to be elucidated. Before Bauhinia championii (Benth.) Benth. is further developed as an agent for treating OA, its underlying molecular mechanism requires investigation.

The cell cycle is composed of four different stages; the G1, S, G2 and M phases. Preparation for DNA synthesis occurs during the G1 phase, DNA synthesis is carried out during the $\mathrm{S}$ phase, preparation for mitosis occurs in the G2 phase and mitosis is achieved in the $M$ phase. The amount of DNA in a cell changes during the cell cycle, allowing the different stages of the cycle to be identified by analyzing the DNA content. The DNA content is $2 \mathrm{~N}$ in the G0 and G1 phases. Following DNA synthesis in the $\mathrm{S}$ phase, DNA content becomes $4 \mathrm{~N}$ in the $\mathrm{G} 2$ and $\mathrm{M}$ phases $(26,27)$. Flow cytometric analysis, which measures the DNA content of cells, is more sensitive to the changes during the cell cycle compared with the MTT method. In the present study, cell cycle distribution was carried out using flow cytometry. The results showed that after BCBP treatment, the G0/G1 ratio was reduced and the $\mathrm{S}$ ratio increased, indicating that BCBP treatment promotes cell proliferation by enhancing G1 phase entry and accelerating the $\mathrm{G} 1 / \mathrm{S}$ transition.

G1/S and G2/M are two important checkpoints regulating stage transition and cell cycle progression. Stage transitions in the cell cycle are controlled by interactions among the molecules of the cyclin-CDK-CDK inhibitor (CKI) axis. In this system, cyclins interact with CDKs to positively regulate their activity $(28,29)$. Cyclin D1, CDK4 and CDK6 are important in the G1/S transition of the cell cycle. The mRNA and protein expression levels of cyclin D1, CDK4 and CDK6 in chondrocytes were detected using RT-PCR and western blot analysis, respectively. The results showed that BCBP treatment effectively enhanced the mRNA and protein levels of cyclin D1, CDK4 and CDK6. In agreement with these results, flow cytometric analysis showed that the $\mathrm{S}$ ratio increased, while the G1 ratio decreased with BCBP treatment.

In conclusion, the present study has demonstrated that BCBP promotes chondrocyte proliferation by accelerating the G1/S transition and upregulating the expression of cyclin D1, CDK4 and CDK6. These results suggest that BCBP is a potential novel therapeutic agent for the treatment of knee OA.

\section{Acknowledgements}

This study was supported by the National Natural Science Foundation of China (no. 81072826), the Natural Science Foundation of Fujian Province (no. 2010Y0032) and the Chen Keji Integrative Medicine Developmental Foundation (no. CKJ2011003).

\section{References}

1. Wattanachai T, Yonemitsu I, Kaneko S and Soma K: Functional lateral shift of the mandible effects on the expression of ECM in rat temporomandibular cartilage. Angle Orthod 79: 652-659, 2009.

2. Gentili $\mathrm{C}$ and Cancedda R: Cartilage and bone extracellular matrix. Curr Pharm Des 15: 1334-1348, 2009.

3. Brondello JM, Philipot D, Djouad F, Jorgensen C and Noël D: Cellular Senescence is a Common Characteristic Shared by Preneoplasic and Osteo-Arthritic Tissue. Open Rheumatol J 4: 10-14, 2010.

4. Grimmer C, Balbus N, Lang U, Aigner T, Cramer T, Müller L, Swoboda B and Pfander D: Regulation of type II collagen synthesis during osteoarthritis by proly-4-hydroxylases: possible influence of low oxygen levels. Am J Pathol 169: 491-502, 2006.

5. Tsuji K, Bandyopadhyay A, Harfe BD, Cox K, Kakar S, Gerstenfeld L, Einhorn T, Tabin CJ and Rosen V: BMP2 activity, although dispensable for bone formation, is required for the initiation of fracture healing. Nat Genet 38: 1424-1429, 2006.

6. Sherr CJ and Roberts JM: Living with or without cyclins and cyclin-dependent kinases. Genes Dev 18: 2699-2711, 2004.

7. Planas-Silva MD and Weinberg RA: The restriction point and control of cell proliferation. Curr Opin Cell Biol 9: 768-772, 1997.

8. Zetterberg A and Larsson O: Kinetic analysis of regulatory events in G1 leading to proliferation or quiescence of Swiss 3T3 cells. Proc Natl Acad Sci USA 82: 5365-5369, 1985.

9. Zhang M, Xie R, Hou W, Wang B, Shen R, Wang X, Wang Q, Zhu T, Jonason JH and Chen D: PTHrP prevents chondrocyte premature hypertrophy by inducing cyclin-D1-dependent Runx2 and Runx3 phosphorylation, ubiquitylation and proteasomal degradation. J Cell Sci 122: 1382-1389, 2009.

10. Newman DJ, Cragg GM and Snader KM: The influence of natural products upon drug discovery. Nat Prod Rep 17: 215-234, 2000.

11. Tzianabos AO: Polysaccharide immunomodulators as therapeutic agents: structural aspects and biological function. Clin Microbiol Rev 13: 523-533, 2000.

12. Paulsen BS: Plant polysaccharides with immunostimulatory activities. Curr Org Chem 5: 939-950, 2001.

13. Wasser SP: Medicinal mushrooms as a source of antitumor and immunomodulating polysaccharides. Appl Microbiol Biotechnol 60: 258-274, 2002.

14. Xu R, Ye H, Sun Y, Tu Y and Zeng X: Preparation, preliminary characterization, antioxidant, hepatoprotective and antitumor activities of polysaccharides from the flower of tea plant (Camellia sinensis). Food Chem Toxicol 50: 2473-2480, 2012.

15. Zhang BZ, Yan PS, Chen $\mathrm{H}$ and He J: Optimization of production conditions for mushroom polysaccharides with high yield and antitumor activity. Carbohydr Polym 87: 2569-2575, 2012.

16. Hua Y, Gao Q, Wen L, Yang B, Tang J, You L and Zhao M: Structural characterisation of acid- and alkali-soluble polysaccharides in the fruiting body of Dictyophora indusiata and their immunomodulatory activities. Food Chem 132: 739-743, 2012.

17. Sui Z, Gizaw Y and BeMiller JN: Extraction of polysaccharides from a species of Chlorella. Carbohydr Polym 90: 1-7, 2012. 
18. The Ministry of Science and Technology of the People's Republic of China: Guidance Suggestions for the Care and Use of Laboratory Animals. Beijing, China, 2006.

19. Li X, Du M, Liu X, et al: Millimeter wave treatment promotes chondrocyte proliferation by upregulating the expression of cyclin-dependent kinase 2 and cyclin A. Int J Mol Med 26: 77-84, 2010.

20. Machida YJ, Hamlin JL and Dutta A: Right place, right time, and only once: replication initiation in metazoans. Cell 123: 13-24, 2005.

21. Nigg EA: Mitotic kinases as regulators of cell division and its checkpoints. Nat Rev Mol Cell Biol 2: 21-32, 2001.

22. Heinegård D, Bayliss MT and Lorenzo P: Biochemistry and metabolism of normal and osteoarthritic cartilage. In: Osteoarthritis. Brandt KD, Doherty M and Lohmander LS (eds). Oxford University Press, New York, NY, pp74-84, 1998.

23. Pritzker KPH: Pathology of osteoarthritis. In: Osteoarthritis. Brandt KD, Doherty M and Lohmander LS (eds). Oxford University Press, New York, NY, pp50-61, 1998.

24. Kim HA and Blanco FJ: Cell death and apoptosis in osteoarthritic cartilage. Curr Drug Targets 8: 333-345, 2007.
25. Chan BY, Fuller ES, Russell AK, et al: Increased chondrocyte sclerostin may protect against cartilage degradation in osteoarthritis. Osteoarthritis Cartilage 19: 874-885, 2011.

26. Hwang SG, Song SM, Kim JR, Park CS, Song WK and Chun JS: Regulation of type II collagen expression by cyclin-dependent kinase 6, cyclin D1, and p21 in articular chondrocytes. IUBMB Life 59: 90-98, 2007.

27. Li TF, Chen $\mathrm{D}, \mathrm{Wu} \mathrm{Q}$, et al: Transforming growth factor- $\beta$ stimulates cyclin D1 expression through activation of $\beta$-catenin signaling in chondrocytes. J Biol Chem 281: 21296-21304, 2006.

28. Susaki E, Nakayama K, Yamasaki L and Nakayama KI: Common and specific roles of the related CDK inhibitors p27 and p57 revealed by a knock-in mouse model. Proc Natl Acad Sci USA 106: 5192-5197, 2009.

29. Cheng A and Solomon MJ: Speedy/Ringo C regulates S and G2 phase progression in human cells. Cell Cycle 7: 3037-3047, 2008. 